\title{
Toxin-B-Antikörper senkt Rezidivrate bei Clostridium-difficile-Infektion
}

In einer großen klinischen Studie konnte eine Behandlung mit einem Antikörper gegen das Toxin B von Clostridium difficile die Rezidivrate der Infektion signifikant senken. Ein Antikörper gegen Toxin A war bei dieser Indikation hingegen ohne Wirkung.

Hintergrund und Fragestellung: Rezidivierende Verläufe nach einer initial erfolgreichen Behandlung einer Clostridium difficile-Infektion (CDI) treten in bis zu 25\% der Betroffenen auf. Die Wahl des Antibiotikums, das zur Behandlung eingesetzt wird, kann dieses Rezidivrisiko beeinflussen. Doch auch bei einer geeigneten Auswahl treten Rezidive auf. In Anbetracht der mit einer CDI verbundenen Morbidität und Mortalität, insbesondere bei Risikopatienten, ist das Vermeiden von Rezidiven ein wünschenswertes Ziel. Neben verschiedenen Antibiotika wurden nun erstmals Antikörper gegen die Clostridientoxine A und B, die zusätzlich zur Standardtherapie verabreicht wurden, in einer klinischen Studie geprüft.

Patienten und Methoden: In zwei nahezu identischen, multizentrischen, placebokontrollierten, randomisierten klinischen Studien wurden 2.655 erwachsene Patienten eingeschlossen. Dabei wurden sowohl Patienten mit einer Erstinfektion als
Wilcox MH et al. Bezlotoxumab for Prevention of Recurrent Clostridium difficile Infection. New Engl J Med 2017; 376: $305-17$ auch Patienten mit einer wiederkehrenden CDI berücksichtigt. Zusätzlich zur antibiotischen Therapie, die vom einschließenden Arzt ent- sprechend der klinischen Situation frei gewählt wurde, erhielten die Patienten in den MODIFY-I- und -II-Studien eine einmalige Infusion mit einem Toxin-B-Antikörper (Bezlotoxumab), mit einem Toxin-A-Antikörper (Actoxumab), mit beiden Antikörpern oder eine Placeboinfusion. Den Behandlungsarm mit dem Toxin-A-Antikörper Actoxumab allein gab es nur in der MODIFY-I-Studie. Die verabreichte Dosis betrug für jeden Antikörper jeweils 10 mg pro Kilogramm Körpergewicht.

Ergebnisse: Als primärer Endpunkt wurde eine Rekurrenz innerhalb von zwölf Wochen nach Behandlungsbeginn der CDI gewählt. In der MODIFY-I-Studie trat nach Infusion des ToxinA-Antikörpers Actoxumab bei $26 \%$ Patienten (im Vergleich Placebo $28 \%$ ) eine Rekurrenz auf, sodass dieser Arm in der MODIFY-II-Studie nicht weiter verfolgt wurde. Bei den Patienten, die eine Infusion mit dem Toxin-B-Antikörper Bezlotoxumab erhielten, trat in der gepoolten Analyse in $17 \%$ eine Rekurrenz auf. Dies war signifikant niedriger verglichen mit der gepoolten Placebogruppe $(27 \%, \mathrm{p}<0,001)$. Die Ergebnisse in der Gruppe der Patienten, die eine Infusion mit beiden Antikörpern erhielt, waren den Ergebnissen in der Placebogruppe ebenso signifikant überlegen $(15 \%, p<0,001)$, eine signifikante Steigerung gegenüber der alleinigen Toxin-B-Antikörpergabe fand sich aber nicht. Die number needed to treat lag bei 10, in der Patientengruppe über 65 Jahre bei 6 .

Die häufigsten beobachteten Nebenwirkungen in der Studie waren Übelkeit und Diarrhö. Die Nebenwirkungen unterschieden sich in den Placebo- und Verumgruppen aber nicht signifikant.

\section{- Kommentar von Martin Storr, Starnberg}

\section{Ein spannender neuer Ansatz}

Antikörper gegen die Clostridium-difficile-Therapie sind nicht für die alleinige Behandlung der Infektion geeignet. Der klinischen Prüfung folgend ist der Toxin-B-Antikörper, in Kombination mit einer Standardtherapie, ein interessanter zusätzlicher Ansatzpunkt, der die hohe Rezidivrate der CDI zu reduzieren vermag. Die Rezidivrate wurde um zirka $40 \%$ gesenkt.

Der Wirkmechanismus der hier eingesetzten Therapie begründet sich in einer neutralisierenden Wirkung des Clostridium-difficile-Toxin-B durch den Antikörper Bezlotoxumab und damit einer Reduktion von Darmwandschädigung und Entzündungsreaktion. Bei Toxin A zeigt sich trotz eines vergleichbaren Wirkmechanismus kein klinischer Vorteil. Eine einmalige Infusion des Antikörpers ist aufgrund der langen Halbwertszeit von zirka 19 Tagen ausreichend. Dies ist für einen möglichen zukünftigen Einsatz vorteilhaft, da die Patienten

nicht durch zusätzliche regelmäßige Medikamentengaben belastet werden.

Zahlreiche Publikationen haben uns in letzter Zeit dahingehend sensibilisiert, dass die Zahl der CDI steigt, dass die Rate der Rezidive hoch ist, und dass dies insbesondere bei Risikopatienten zu einer gesteigerten Morbidität und Mortalität führt. Der Begriff Risikopatient wird in diesem Zusammenhang nicht immer gleich verwendet, Risikofaktoren wie ein Alter > 65 Jahre, Co-Morbiditäten, CDI in der Vergangenheit und ein schwerer Verlauf sind aber klinisch gut nachvollziehbar. Ein möglicher zukünftiger Zulassungstext wird bei der Entscheidung helfen, ob und in welcher Situation ein Einsatz von Bezlotoxumab angebracht sein könnte. Patienten mit schweren Verläufen, Risikofaktoren oder hochvirulenten Stämmen wie dem Ribotyp 027, erscheinen als eine gut geeignete Patientengruppe. 